\title{
Engineered Generation of Human Natural Killer Cells From Different Somatic Cells Subjected to Physical Reprogramming
}

\section{Na Song ( $564115820 @ q q . c o m$ )}

Sichuan University State Key Laboratory of Biotherapy https://orcid.org/0000-0003-1094-4091

Jian chuan Hu

Sichuan University State Key Laboratory of Biotherapy

Chao Su

Sichuan University State Key Laboratory of Biotherapy

Xian cheng Chen

Sichuan University State Key Laboratory of Biotherapy

\section{Research}

Keywords: pluripotent embryonic stem cells, physical dynamic culture, NK cells, cytokine

Posted Date: April 22nd, 2021

DOI: https://doi.org/10.21203/rs.3.rs-426992/v1

License: (a) (i) This work is licensed under a Creative Commons Attribution 4.0 International License. Read Full License 


\section{Abstract}

Background: As part of the innate immune system, natural killer (NK) cells can directly kill virus-infected cells and malignant cells without pre-immunization. NK cell-based immunotherapy has attracted tremendous attention as a practical treatment for cancer and other diseases. In order to provide unlimited "off-the-shelf" NK cells to serve many recipients, we designed and demonstrated an overall manufacturing scheme for mass production of NK cells from physically reprogrammed somatic cells.

Methods: Different somatic cells would be reprogrammed into induced pluripotent stem cells (iPSCs) by physical-dynamic suspension culture system, which was non-viral, non-integrated, non-compoundinduced, feeder-free, and serum-free. The pluripotency of iPSCs was assessed by immunofluorescence, flow cytometry analysis, western blot, and teratoma assay. By using a combination of cytokines, iPSCs would be further differentiated into NK cells. Immunofluorescence, flow cytometry analysis, and lactate dehydrogenase (LDH) release method were performed to evaluate the characteristics of NK cells.

Results: Starting from human somatic cells, we obtained iPSCs in the form of spherical embryoids (SEs) through physical reprogramming. iPSCs were similar to embryonic stem cells (ESCs) in their characteristics, including morphology, expression of pluripotency-associated markers, and teratoma formation. NK cells were efficiently generated from SEs by a combination of cytokines under feeder-free conditions. SEs-NK cells share similar phenotypic and functional characteristics with human NK cells, including morphology, cell surface markers, and cytotoxicity.

Conclusions: We have not only established a practical approach for large-scale production of universal NK cells, but also provided a platform for generating good manufacturing practice (GMP)-compliant iPSCs through physical dynamics, thus suggesting that the physical reprogramming will become a more promising strategy for future biotherapies.

\section{Background}

As important effector lymphocytes of the innate immune system, NK cells form the first line of defense against pathogens or stressed / cancerous host cells, and are the bridge between innate and adaptive immunity. Mature NK cells can directly kill virus-infected cells and malignant cells, which is different from the $T$ cell response that is restricted by the major histocompatibility complex $(\mathrm{MHC})$ and requires prior sensitization [1]. In addition, NK cells play an important role in tumor immunosurveillance and the maintenance of immune homeostasis. Therefore, more and more researches have focused on NK cellrelated immunotherapy. It has been demonstrated that NK cell-based immunotherapy is a promising cellular immunotherapy to revitalize and improve the function of the immune system [2]. The main obstacle to the clinical application of NK cell-related immunotherapy is how to obtain high-yield and highquality NK cells. Currently, NK cells are mainly obtained from peripheral blood, umbilical cord blood (UCB), NK cell lines, and human pluripotent stem cells (hPSCs), including ESCs and iPSCs [3-5]. Compared with the low distribution rate of NK cells in peripheral blood, the poor function of UCB-derived NK cells, and the 
clinical safety considerations of NK cells expanded from NK cell line, iPSCs serve as a potential source of NK cells due to their characteristics of self-renewal and multipotent differentiation.

iPSCs can be produced by reprogramming of somatic cells through overexpression of Sox2, Oct4, c-Myc, and KIf4, and exhibit characteristics of ESCs, including self-renewal and differentiation potential [6-10]. It is reported that IPSC can be obtained from skin fibroblasts, blood cells, keratinocytes, and epithelial cells isolated from urine, and will not bear the ethical issues of ESCs [11-13]. iPSCs have been widely applied to regenerative medicine, drug screening, and disease modeling [14]. In recent years, the progress of reprogramming research has caused significant changes in iPSCs technology, including reprogramming factors, vectors, and culture conditions $[15,16]$. From the initial integrated gene vectors to relatively safe and reliable non-integrated gene vectors, from feeder culture methods to suspension culture methods without heterogenous substance, iPSCs technology gradually tends to simplify, procedural and largescale $[15,16]$.

NK cells derived from iPSCs are mature cells that can secrete cytokines and have cytotoxic effects on malignant cells in vivo and in vitro $[5,17,18]$. Existing protocols are not suitable for large-scale production of NK cells from iPSCs, such as CD34+ hematopoietic progenitor cell enrichment, feeder layer (mouse stromal cells), or long-term expansion $[1,5,18]$. In order to eliminate xenogeneic cells, simplify the culture protocol, and provide unlimited "off-the-shelf" NK cells, we designed a two-step manufacturing protocol to generate NK cells from physically reprogrammed somatic cells. Starting from somatic cells, iPSCs in the form of SEs were generated through physical reprogramming that does not require gene integration, compound induction, feeder layer, and serum. Then functional and scalable NK cells were generated from SEs through our original protocol, which excluded $\mathrm{CD} 34^{+}$cell enrichment and feeder layer. We have not only established a practical method to produce a large number of universal NK cells, but also provided a platform for generating good manufacturing practice (GMP)-compliant iPSCs through physical reprogramming, which simplifies the reprogramming process of somatic cells and saves the time and cost of iPSCs production.

\section{Methods}

\section{Cell lines}

LO2 (human hepatic epithelial cell line) and HOSEC (human ovarian surface epithelial cell line) were obtained from Procell Life Science \& Technology Co., LTD, and were cultured in DMEM/F12 medium. WPMY-1 (human prostate stromal cell line) and K562 (human chronic myeloid leukemia cell line) were obtained from State key laboratory of biotherapy/Collaborative Innovation Center for Biotherapy of Sichuan Province which was tested and authenticated. WPMY-1 cells were cultured in DMEM medium. K562 cells were cultured in RPMI- 1640 medium. All the media were added with $10 \%$ fetal bovine serum (FBS) (Gibco) and 1\% penicillin-streptomycin.

\section{Dynamic Generation of SEs}


To generate SEs from somatic cells, LO2, HOSEC, and WPMY-1 cells were seeded into $100 \mathrm{~mm}$ petri dishes (Corning) and cultured in serum-free DMEM/F12 medium. At $37{ }^{\circ} \mathrm{C}$ and $5 \% \mathrm{CO}_{2}$, LO2 and HOSEC cells were incubated statically for 10-15 minutes and WPMY-1 cells were incubated statically for 50-60 minutes. Then cell suspensions on dishes were subjected to physical-dynamic culture at about 100 swings/min in swinging-rocking-bed with a humidified atmosphere consisting of $5 \% \mathrm{CO}_{2}$. Cells were maintained under dynamical condition for at least 7 9 days to ensure the formation of SEs in the petri dishes. Half of medium was replaced daily under dynamic suspension processes.

\section{NK cell differentiation from SEs (SEs-NK)}

The SEs were suspended in the NK cell induction medium in a 24-well plate. NK cell induction medium consisted of DMEM/F12, $10 \mathrm{ng} / \mathrm{ml}$ interleukin Interleukin (IL)-3 (Novoprotein Scientific Inc.), 20ng/ml IL-7 (Novoprotein Scientific Inc.), 20ng/ml IL-15 (Novoprotein Scientific Inc.), 20ng/ml stem cell factor (SCF, Novoprotein Scientific Inc.), 20ng/ml Fms-related tyrosinekinase3 ligand (Flt3-L, Novoprotein Scientific Inc.), $10 \% \mathrm{FBS}$, and $1 \%$ penicillin-streptomycin. Cells were then cultured in an incubator at $37^{\circ} \mathrm{C}$ and $5 \% \mathrm{CO}_{2}$ for at least $4 \sim 5$ weeks to ensure the formation of SEs-NK cells. Half of the medium was replaced with fresh medium every 3 days.

\section{Immunofluorescence staining of SEs and SEs-NK cells}

The SEs were fixed with methanol at room temperature for 5 minutes. After washing with PBS, SEs were permeabilized with $0.3 \%$ Triton X-100 (Biosharp) in PBS at room temperature for 1 hour. PBS with $5 \%$ bovine serum albumin (BSA) was then added to block for 30 minutes at room temperature. Then, SEs were incubated with primary antibodies against NANOG (1:200, Cell Signaling), OCT4 (1:200, Cell Signaling), SOX2 (1:200, Cell Signaling), stage-specific embryonic antigen (SSEA) 3 (1:400, R\&D Systems), and SSEA4 (1:400, R\&D systems) overnight at $4{ }^{\circ} \mathrm{C}$. After gently washing three times with PBS, SEs were incubated with Alexa647-conjugated mouse anti rabbit IgG (1:100, Bioss), fluorescein isothiocyanate (FITC)-conjugated rabbit anti rat IgM (1:100, Bioss), and FITC-conjugated goat anti mouse IgG (1:100, Bioss) for 1 hour at room temperature in the dark. Immunofluorescence images were observed and collected with a fluorescence microscope (Nikon).

The SEs-NK cells were fixed with methanol at room temperature for 5 minutes. After blocking with $5 \%$ BSA in PBS for 30 minutes at room temperature, cells were incubated with anti-CD57 (1:400, Merck) antibody overnight at $4{ }^{\circ} \mathrm{C}$. Cells were stained with FITC-conjugated goat anti mouse IgG (1:100, Bioss) for 1 hour at room temperature in the dark after through washing with PBS. Immunofluorescence images were observed and collected with a fluorescence microscope (Nikon).

\section{Flow cytometric analysis of SEs and SEs-NK cells}

The SEs were resuspended in PBS contained 5\% BSA for 30 minutes at room temperature. Then SEs were stained with anti-NANOG (1:200, Cell Signaling), OCT4 (1:200, Cell Signaling), SOX2 (1:200, Cell Signaling), SSEA3 (1:400, R\&D Systems), and SSEA4 (1:400, R\&D systems) overnight at $4{ }^{\circ} \mathrm{C}$. After 
washing three times with PBS, SEs were incubated with Alexa647-conjugated mouse anti rabbit IgG (1:100, Bioss), FITC-conjugated rabbit anti rat IgM (1:100, Bioss), and FITC-conjugated goat anti mouse

IgG (1:100, Bioss) for 1 hour at room temperature in the dark. SEs without the primary and the secondary antibody were used as control groups, and samples without the primary antibody were run in parallel.

The SEs-NK cells were blocked with $5 \%$ BSA in PBS for 30 minutes at room temperature. Then cells were stained with anti-CD57 (1:400, Merck) antibody overnight at $4{ }^{\circ} \mathrm{C}$. After washing three times with PBS, cells were incubated with FITC-conjugated goat anti mouse IgG (1:100, Bioss) for 1 hour at room temperature in the dark. Somatic cells (LO2, HOSEC, and WPMY-1 cells) were used as controls, and samples without the primary antibody were run in parallel.

The stained cells were analyzed with NOVO flow cytometer (ACEA Biosciences) using NovoExpress software according to the manufacturer's instructions. The cross-reactivity of these antibodies was considered negligible.

\section{Protein Extraction and Western Blot}

The SEs were lysed in RIPA buffer with protease inhibitor cocktail on ice for $30 \mathrm{~min}$ and quantified with a bicinchoninic acid protein assay kit (Beyotime). Protein fractions were separated by $12 \%$ sodium dodecyl sulfate-polyacrylamide gel electrophoresis and transferred to polyvinylidene difluoride membranes $(0.22$ $\mu \mathrm{m}$, Millipore). The membranes were blocked with $5 \%$ defatted milk and incubated with specific primary antibodies against NANOG (1:1000, BD Biosciences), OCT3/4 (1:1000, BD Biosciences), SOX2 (1:1000, BD Biosciences), or TUBULIN (1:5000, Proteintech) overnight at $4{ }^{\circ} \mathrm{C}$ and then incubated with horseradish peroxidase-conjugated secondary antibody (1:5000, ZSGB-BIO) at room temperature for 1 hour. The signal was revealed using Luminata Crescendo Western HRP substrate reagent (Millipore) and was detected using iBright Western Blot imaging system (Thermo Fisher Scientific). ImageJ software was used to analyze the integrated density (IntDen) of protein bands detected in the Western blot.

\section{Teratoma assay}

To assess the in vivo pluripotency of SEs, SEs were injected subcutaneously into the groin of 4-week-old male severe combined immunodeficiency (SCID) mice (Beijing HFK Bioscience Co., LTD). Around ten weeks after injection, SCID mice were sacrificed and tumors were dissected. Snap-frozen tumor sections were stained with hematoxylin and eosin (H\&E) for histological analysis.

\section{Karyotyping}

Cells were treated with $0.6 \mu \mathrm{g} / \mathrm{ml}$ colchicine for 4 hours and harvested. After hypotonic treatment with $0.075 \mathrm{M} \mathrm{KCl}$ at $37^{\circ} \mathrm{C}$ for 10 minutes, cells were fixed three times with 3:1 mixture of methanol: acetic acid. Cells were then dropped on a pre-chilled glass slide to obtain metaphase chromosome spreading. Chromosomes were stained with Giemsa (Solarbio) and imaged with a microscope.

\section{Cytotoxicity assay}


LDH release method (LDH Cytotoxicity Assay) was used to detect the direct cytotoxicity of derived SEs-

NK cells to target cells. SEs-NK cells were used as the effector cells, and K562 cells were used as the target cells in the cytotoxicity assay. The effector-target ratios (E: T) were 20:1, 50:1, 80:1, and 100:1. Cytotoxicity was determined by LDH release from cells according to the manufacturer's instructions (Beyotime). The resultant LDH release was analyzed using an ELISA plate reader (Thermo Scientific). The cytotoxicity was calculated by the following formula:

\section{LDH release (Experimental - Effector spontaneous Cytotoxicity $(\%)=\frac{- \text { target spontaneous }+ \text { Bankground correction) }}{\text { LDH release (Target maximum }- \text { Target spontaneous }} \times 100 \%$
- Volume correction + Background correction)}

\section{Statistical analysis}

Data were analyzed using GraphPad Prism 8 (GraphPad Software). Student's $t$-tests and one-way analysis of variance (ANOVA) were used to analyze between two groups or among multiple groups, respectively. All experiments were repeated three times and data were shown as mean \pm standard error of

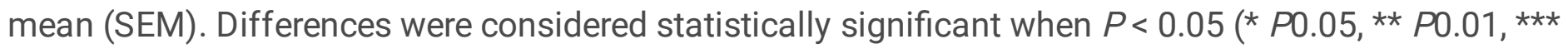
P0.001).

\section{Results}

\section{Adoption of dynamic suspension culture system for generating SEs}

We have developed a highly efficient two-step protocol to produce NK cells from physically reprogrammed somatic cells (Fig. 1a). In order to generate SEs, LO2, HOSEC, and WPMY-1 cells were harvested and transferred to a dynamic suspension culture system without integration, feeder layer, and serum. The morphological changes of cells after dynamic suspension culture were shown in Fig. 1b. Cell aggregates were observed in LO2 and HOSEC cells on day 1, and SEs with irregular shapes appeared on day 3 5. After 5 7 days, SEs with consistent sphere morphology can be formed in both LO2 and HOSEC cells, and the size continued to increase. After 9 days, SEs derived from LO2 (L-SEs) and HOSEC (H-SEs) were in the form of homogeneous spheres wrapped by membrane. SEs derived from WPMY-1 cells (WSEs) were formed on day 7, which was shorter than L-SEs and H-SEs. After 2 3 days, the sphere model of W-SEs was formed rapidly, and the size continued to increase. After 7 days, W-SEs were in the form of homogeneous spheres wrapped by membrane. These results indicate that somatic cells can be efficiently reprogrammed into SEs by dynamic suspension culture system. SEs were compact spheres with clear borders.

During the dynamic suspension culture process, the diameters of L-SEs, H-SEs, and W-SEs increased from $36.682 .49 \mu \mathrm{m}, 34.631 .98 \mu \mathrm{m}$, and $49.414 .00 \mu \mathrm{m}$ to $97.200 .89 \mu \mathrm{m}, 95.431 .56 \mu \mathrm{m}$, and $98.209 .21 \mu \mathrm{m}$, respectively (Fig. 1c). At the beginning and end of the dynamic suspension culture, the average diameters of SEs derived from somatic cells were $40.244 .62 \mu \mathrm{m}$ and $96.940 .81 \mu \mathrm{m}$, respectively (see Additional file 
1). During the dynamic suspension culture process, the diameters of L-SEs, H-SEs, and W-SEs increased $2.670 .18,2.770 .16$, and 1.990 .05 -fold, respectively, and the average diameter of somatic cell-derived SEs increased 2.480 .25 -fold (see Additional file 1). These data show that at the end of the culture, the diameters of the three somatic cell-derived SEs are similar and significantly increased compared to the beginning of the dynamic suspension culture (Fig. 1c).

From $1.220 .0510^{6}$ LO2, $0.980 .0610^{6}$ HOSEC, and $1.580 .0610^{6}$ WPMY-1 cells, we obtained $0.540 .1210^{6}$ L-SEs, $0.230 .0610^{6} \mathrm{H}$-SEs, and $0.260 .0610^{6} \mathrm{~W}$-SEs, respectively (see Additional file 2). On average, we obtained $0.340 .1010^{6}$ SEs from $1.260 .1710^{6}$ somatic cells (see Additional file 2). These data indicate that the total number of cells changes during the formation of SEs.

\section{SEs express pluripotency-associated markers}

The pluripotency-associated markers of SEs were identified by classical assays. Immunofluorescence staining showed that L-SEs, H-SEs, and W-SEs expressed pluripotency-associated markers, such as nuclear markers NANOG, SOX2, OCT4, and surface markers SSEA3 and SSEA4 (Fig. 2a-c).

Two-color flow cytometry analysis demonstrated that L-SE, H-SEs, and W-SEs were positive for NANOG, SOX2, OCT4, SSEA-3, and SSEA-4 (Fig. 3a). Although the expression levels of NANOG, SOX2, OCT4, SSEA3 , and SSEA4 were higher than those of the control group (see Additional file 3), their expression levels in L-SE, H-SE, and W-SE were comparable (Fig. 3b). The percentage of cells expressing pluripotency-related markers was similar in L-SEs, H-SEs, and W-SEs (33.28 5.57\%, $35.213 .42 \%$ and $32.668 .41 \%$ ), and the average percentage of cells expressing pluripotency-related markers was $33.720 .77 \%$ in SEs derived from somatic cells (see Additional file 3). Generation of iPSCs from somatic cells is affected by many factors, such as the initial cell type, reprogramming methods, and culture conditions. Generally, the reprogramming efficiency varies in the range of $10 \%$, and it takes about 12-30 days [11]. In our study, the reprogramming efficiency was comparable for LO2, HOSEC, and WPMY-1(14.93 5.06\%, $7.901 .03 \%$ and $5.481 .84 \%$ ). We obtained an average reprogramming efficiency of $9.442 .83 \%$ when our integration-free, feeder-free, and serum-free dynamic suspension culture system was adopted for the reprogramming of somatic cells (Fig. 3c).

The western blot experiments indicated that NANOG, SOX2, and OCT3/4 were expressed in L-SEs, H-SEs, and W-SEs cells, and the expression of OCT3/4 was higher in the three somatic cell-derived SEs (Fig. 4a and see Additional file 4). W-SEs expressed NANOG protein at higher levels compared to L-SEs and H-SEs. L-SEs expressed SOX2 protein at higher levels compared to H-SEs and W-SEs. There was no significant difference in the protein level of OCT3/4 among the three somatic cell-derived SEs (Fig. 4b). Taken together, the results of immunofluorescence staining, flow cytometry analysis, and western blot analysis indicate that SEs generated in the dynamic suspension culture system express pluripotent-associated markers.

\section{Teratoma formation from SEs with normal karyotype}


To test pluripotency in vivo, we transplanted SEs subcutaneously into the groin of immunodeficient (SCID) mice. Ten weeks after injection, we observed tumor formation. Histological examination showed that the tumor contained various tissues (Fig. 5a), including thyroid tissues (endoderm), chondrocyte (mesoderm), and neural tissues (ectoderm). Finally, L-SEs, H-SEs, and W-SEs displayed normal karyotypes (Fig. 5b).

In summary, our dynamic suspension culture system allows us to efficiently generate integration-free iPSCs (in the form of SEs) from somatic cells. SEs are characterized by classic assays, including contribution to all three germ layers in vivo. More importantly, all classic assays were performed after 7 9 days of dynamic suspension culture suggesting that our protocol can provide high-quality iPSCs in a short time.

\section{Directed differentiation of SEs into NK cells}

We next examined whether SEs could be induced to directly differentiate into NK cells without employing $\mathrm{CD} 34^{+}$cell enrichment and feeder. The protocol of NK cell induction is summarized in Fig. 6a. We transferred L-SEs, H-SEs, and W-SEs to 24-well plates and maintained them under differentiation conditions for 4 5 weeks. Cells spread after 3 days, and a large number of suspension cells were observed after 30 days (Fig. 6b). Morphologically, L-SEs, H-SEs, and W-SEs could differentiate into small, round, suspended, and resembled lymphoid cells under cytokines conditions.

From $0.270 .0610^{6}$ L-SEs, $0.110 .0310^{6} \mathrm{H}$-SEs, and $0.130 .0310^{6} \mathrm{~W}$-SEs, we obtained $0.340 .0610^{6} \mathrm{~L}$-SEsNK, $0.220 .1110^{6} \mathrm{H}$-SEs-NK, and $1.100 .2110^{6} \mathrm{~W}$-SEs-NK cells, respectively (see Additional file 5). On average, we obtained $0.550 .2710^{6} \mathrm{SEs}-\mathrm{NK}$ cells from $0.170 .0510^{6} \mathrm{SEs}$ (see Additional file 5). The total number of L-SEs-NK, H-SEs-NK, and W-SEs-NK cells increased about 1.40 0.25, 2.12 1.08, and 9.28 1.78fold, and the average total number of SEs-NK cells increased about 4.26 2.52-fold (see Additional file 5). These data indicate that the total number of cells changes during the formation of SEs-NK cells.

\section{Surface marker analysis of SEs-NK cells}

The expression of CD57 on NK cells has been demonstrated by a large number of studies [19-24]. More recently, it has been established that CD57 expression defines functionally mature sub-populations of NK cells [25-27]. Immunofluorescence staining showed that CD57 was expressed on L-SEs-NK, H-SEs-NK, and W-SEs-NK cells, but not on LO2, HOSEC, and WPMY-1 cells (Fig. 7a).

Flow cytometry showed that the expression level of CD57 on L-SEs-NK, H-SEs-NK, and W-SEs-NK cells was higher than that on LO2, HOSEC, and WPMY-1 cells (Fig. 7b and see Additional file 6). For SEs-NK cells, we found that a majority of L-SEs-NK and H-SEs-NK cells expressed CD57 (see Additional file 6). Compared to somatic cells, SEs-NK cells included a significantly higher proportion of NK cells expressing CD57 (see Additional file 6). These results indicate that SEs-NK cells generated in this study are CD57 ${ }^{+}$ NK cells, indicating that they are highly mature and terminally differentiated NK cells. 


\section{Cytotoxic activity of SEs-NK cells}

Cytotoxicity is an important functional feature of NK cells. The cytotoxicity of NK cells can be determined by measuring the percentage of K562 cells lysed by NK cells after incubation. The cytotoxic activities of L-SEs-NK cells against K562 at E: T ratios of 20:1, 50:1, 80:1, and 100:1 were 61.22 6.42\%, 59.40 4.82\%, $63.404 .42 \%$, and $61.835 .10 \%$, respectively, H-SEs-NK cells were 49.72 0.89\%, $50.763 .82 \%, 49.994 .00 \%$, and $51.973 .19 \%$, respectively, and W-SEs-NK cells were $22.543 .53 \%, 33.111 .01 \%, 30.082 .14 \%$, and $28.940 .95 \%$, respectively (Fig. 8a). Similar to the expression of CD57 in SEs-NK cells, L-SEs-NK cells and H-SEs-NK cells had higher ability of K562 cytotoxicity than W-SEs-NK (Fig. 8b). Therefore, through a combination of cytokines, SEs can be directly induced to differentiate into mature NK cells with cytotoxic function, without the need for $\mathrm{CD} 34^{+}$cell enrichment and feeder culture system.

\section{Discussion}

In this study, we showed that NK cells can be generated from physically reprogrammed somatic cells. Inducing the production of iPSCs is the key first step for generating NK cells from somatic cells. It has been observed that reprogramming efficiency and time were $10 \%$ and 12-30 days depending on somatic cell types, reprogramming methods, and culture conditions [11]. Suspension culture systems have been reported for maintenance and expansion of hPSCs (ESCs and iPSCs) maintenance and expansion, such as low-attachment plates, spinner flasks, rotated dishes, Erlenmeyer flasks, and bioreactors [28]. In the suspension culture system, cells are cultured in the form of matrix-free hPSC spheres or

microencapsulated hPSCs, or in the form of hPSCs immobilized on microcarriers [29]. Suspension culture system promotes signal transmission between cells and improves cell crosstalk by simulating the extracellular microenvironment [29]. The media used in 2D feeder-free culture system, including mTeSR1, KSR-based media, and Essential 8, are also used for suspension culture of hPSCs [29]. In addition, some chemical compounds such as MEK inhibitor, Rho-associated kinase (ROCK) inhibitor, and GSK-3 inhibitor are widely used for the maintenance and proliferation of hPSCs in Suspension culture system [29].

Due to the forced expression of transcription factors in somatic cells, iPSCs bypass the ethical restrictions of ESCs [10]. Reprogramming protocols with variations in delivery methods and cell types have been set up to generate iPSCs, and each protocol has its own pros and cons [30]. The process of viral transduction may lead to insertional mutagenesis, and invalid silencing of transcription factors may subsequently render cells immunogenic [31]. Although protein-, mRNA-, and chemical-based reprograming methods can successfully generate iPSCs that are not integrated by any transcription factors, the reprogramming efficiency is very low and the process takes a longer time [32, 33]. Various types of human cells, such as fibroblasts, neural stem cells, mature $\beta$ cells, adipose stem cells, and keratinocytes, have been successfully reprogrammed into iPSCs $[9,10,34-39]$. Studies have shown that the origin of somatic cells (mesenchyme or epithelium) can affects the production of iPSC at the beginning of reprogramming [40]. Epithelial cells and mesenchymal cells are the two major cell types in most animals. The characteristics of epithelial cells are apical-basal axis of polarity, gap junction, and immobility, while 
mesenchymal cells are known for their non-polarity, loose association, and high mobility [41]. Epithelialmesenchymal transition (EMT) and mesenchymal-epithelial transition (MET) have been observed during embryonic development, tumor progression, somatic cell reprogramming, and other multiple cell fate conversions [41]. Recent studies have shown that somatic cell reprogramming is a consecutive process, including EMT and MET, and MET is a crucial step in obtaining pluripotency [42]. Compared with fibroblasts, epithelial cells isolated from ciliary body and urine have higher reprogramming efficiency, making them an excellent candidate for iPSCs generation $[12,43,44]$.

To simply the protocol and improve the efficiency of reprogramming, we have established a feeder- and serum-free dynamic suspension culture system for the efficient generation of iPSCs. Our current method using L02, HOSEC, and WPMY-1 cells as the original somatic cells, does not require exogenous gene for transduction or complicated methods for culture, and achieves reprogramming efficiencies similar to those using other reprogramming methods. Somatic cells were reprogrammed into iPSCs in the form of SEs in the dynamic suspension culture system. On the one hand, this may be related to the existence of undifferentiated cell spheres of iPSCs in the absence of microcarriers. On the other hand, it may be related to a series of continuous EMT and MET during somatic cell reprogramming. The external environment in the dynamic culture system may affect the gene expression of somatic cells, promote the occurrence of EMT and MET, and facilitate the reprogramming of somatic cells into iPSCs.

Promoting differentiation of iPSCs into NK cells is the second step for generating NK cells from somatic cells. As important effector lymphocytes of the innate immune system, NK cells can recognize and directly kill cells lacking MHC class I molecules without pre-immunization [45]. Several studies have found that NK cells can survive up to 20 months after adoptive transplantation and can be well-tolerated [46]. A large number of NK cells have been obtained from peripheral blood mononuclear cells (PBMCs) or NK cell lines through NK cell enrichment or expansion. As with any other manufacturing process that relies on donor cells, it is difficult to consistently generate standard NK cell products from various PBMCs donors using highly variable starting materials. Although NK-92 is the only cell line approved by the US FDA for clinical trials among NK cell lines, these EBV-transfected cells must be irradiated before clinical application to prevent their proliferation in patients [47]. hPSCs are the third source of NK cells. Compared with ESCs, the generation and use of iPSCs (autotherapy and allogeneic therapy) poses fewer ethical problems [48]. Various protocols have been successfully used to generate NK cells from iPSCs, but these protocols often involve procedures that are unsuitable for mass production, e.g., enriching CD $34^{+}$ hematopoietic precursors or forming spin embryoid body (spin-EB) $[1,5,18]$. The process of CD34 ${ }^{+}$ hematopoietic progenitor cell enrichment increases the workload and loses many other hematopoietic progenitor cells that can differentiate into NK cells [48]. The spin-EB protocol is complicated, and the hematopoietic progenitor cells derived from spin-EB are heterogeneous, which may affect the differentiation of NK cells [48].

To establish a practical protocol, we cultured SEs in the presence of a combination of cytokines (IL-3, IL-7, IL-15, SCF, and FLT3-L) to stimulate differentiation and growth of NK cell. Without employing CD34 ${ }^{+}$cell enrichment or spin-EB formation, high-purity, mature, and functional NK cells were generated from SEs. 


\section{Conclusion}

Overall, our data demonstrate an improved protocol to generate mature and functional NK cells from somatic cells by physical reprogramming. Using a step-by-step approach, we were able to transition to a fully defined system suitable for clinical needs. The dynamic suspension culture system provides a simple, safe, and effective reprogramming method without the need for gene integration, compound induction, and feeder. iPSCs obtained via the system are highly accessible and in compliance with GMP. With the continuous optimization of physical dynamic suspension process, our reprogramming efficiency will continue to improve and enhance. NK cells derived from iPSCs are mature and functional, and can be used as "off-the-shelf" products for various recipients. The dynamic suspension culture system not only provides a system for clinical-scale lymphocyte expansion, but also provides a platform for studying human NK cells and iPSCs.

\section{Abbreviations}

NK cell: natural killer cell; iPSCs: induced pluripotent stem cells; LDH: lactate dehydrogenase; SEs: spherical embryoids; ESCs: embryonic stem cells; MHC: major histocompatibility complex; UCB: umbilical cord blood; hPSCs: human pluripotent stem cells; GMP: good manufacturing practice; FBS: fetal bovine serum; rpm: revolutions per minute; SEs-NK: NK cell differentiation from SEs; IL: interleukin; SCF: stem cell factor; Flt3-L: Fms-related tyrosinekinase3 ligand; BSA: bovine serum albumin; SSEA: stage-specific embryonic antigen; FITC: fluorescein isothiocyanate; IntDen: integrated density; SCID: severe combined immunodeficiency; H\&E: hematoxylin and eosin; E: T: effector-target ratios; SEM: standard error of the mean; L-SEs: SEs derived from LO2; H-SEs: SEs derived from HOSEC; W-SEs: SEs derived from WPMY-1 cells; ROCK: Rho-associated kinase; EMT: Epithelial-mesenchymal transition; MET: mesenchymalepithelial transition; PBMCs: peripheral blood mononuclear cells; spin-EB: spin embryoid body

\section{Declarations}

\section{Ethics approval and consent to participate}

The animal study was reviewed and approved by the Ethics Review Committee of Animal Experimentation of Sichuan University.

\section{Consent for publication}

Not applicable.

\section{Availability of data and materials}

All data generated or analyzed during this study are included in this published article and its supplementary information files. 
The authors declare that they have no competing interests.

\section{Funding}

This work was supported by National Natural Science Foundation (30973507) and National Basic Research Program of China (2010CB529900) and High-tech Research and Development Program (2014ZX09101041-002).

\section{Authors' contributions}

The study was designed by XCC and NS. NS carried out the experiments, performed the statistical analyses, and prepared the manuscript. $\mathrm{JCH}$ and CS helped with some of the experiments. All authors have read and approved the final manuscript.

\section{Acknowledgments}

We thank the professors and technologists of National Key Laboratory of Biotherapy, Sichuan University, for their expert technical assistance.

\section{References}

1. Knorr DA, Ni Z, Hermanson D, Hexum MK, Bendzick L, Cooper LJ, et al. Clinical-scale derivation of natural killer cells from human pluripotent stem cells for cancer therapy. Stem Cells Transl Med. 2013;2(4):274-83.

2. Hu W, Wang G, Huang D, Sui M, Xu Y. Cancer Immunotherapy Based on Natural Killer Cells: Current Progress and New Opportunities. Front Immunol. 2019;10:1205.

3. Eguizabal C, Zenarruzabeitia O, Monge J, Santos S, Vesga MA, Maruri N, et al. Natural killer cells for cancer immunotherapy: pluripotent stem cells-derived NK cells as an immunotherapeutic perspective. Front Immunol. 2014;5:439.

4. Ni F, Sun R, Fu B, Wang F, Guo C, Tian Z, et al. IGF-1 promotes the development and cytotoxic activity of human NK cells. Nat Commun. 2013;4:1479.

5. Woll PS, Grzywacz B, Tian X, Marcus RK, Knorr DA, Verneris MR, et al. Human embryonic stem cells differentiate into a homogeneous population of natural killer cells with potent in vivo antitumor activity. Blood. 2009;113(24):6094-101.

6. Okita K, Ichisaka T, Yamanaka S. Generation of germline-competent induced pluripotent stem cells. Nature. 2007;448(7151):313-7.

7. Takahashi K, Yamanaka S. Induction of pluripotent stem cells from mouse embryonic and adult fibroblast cultures by defined factors. Cell. 2006;126(4):663-76.

8. Wernig M, Meissner A, Foreman R, Brambrink T, Ku M, Hochedlinger $K$, et al. In vitro reprogramming of fibroblasts into a pluripotent ES-cell-like state. Nature. 2007;448(7151):318-24. 
9. Yu J, Vodyanik MA, Smuga-Otto K, Antosiewicz-Bourget J, Frane JL, Tian S, et al. Induced pluripotent stem cell lines derived from human somatic cells. Science. 2007;318(5858):1917-20.

10. Takahashi K, Tanabe K, Ohnuki M, Narita M, Ichisaka T, Tomoda K, et al. Induction of pluripotent stem cells from adult human fibroblasts by defined factors. Cell. 2007;131(5):861-72.

11. Malik N, Rao MS. A review of the methods for human iPSC derivation. Methods Mol Biol. 2013;997:23-33.

12. Zhou T, Benda C, Duzinger S, Huang Y, Li X, Li Y, et al. Generation of induced pluripotent stem cells from urine. J Am Soc Nephrol. 2011;22(7):1221-8.

13. Jacobson EF, Tzanakakis ES. Who Will Win: Induced Pluripotent Stem Cells Versus Embryonic Stem Cells for $\beta$ Cell Replacement and Diabetes Disease Modeling? Curr Diab Rep. 2018;18(12):133.

14. Cebrian-Serrano A, Stout T, Dinnyes A. Veterinary applications of induced pluripotent stem cells: regenerative medicine and models for disease? Vet J. 2013;198(1):34-42.

15. Chun YS, Chaudhari P, Jang YY. Applications of patient-specific induced pluripotent stem cells; focused on disease modeling, drug screening and therapeutic potentials for liver disease. Int J Biol Sci. 2010;6(7):796-805.

16. Chang CY, Ting HC, Su HL, Jeng JR. Combining Induced Pluripotent Stem Cells and Genome Editing Technologies for Clinical Applications. Cell Transplant. 2018;27(3):379-92.

17. Ni Z, Knorr DA, Clouser CL, Hexum MK, Southern P, Mansky LM, et al. Human pluripotent stem cells produce natural killer cells that mediate anti-HIV-1 activity by utilizing diverse cellular mechanisms. J Virol. 2011;85(1):43-50.

18. Woll PS, Martin CH, Miller JS, Kaufman DS. Human embryonic stem cell-derived NK cells acquire functional receptors and cytolytic activity. J Immunol. 2005;175(8):5095-103.

19. Brenchley JM, Karandikar NJ, Betts MR, Ambrozak DR, Hill BJ, Crotty LE, et al. Expression of CD57 defines replicative senescence and antigen-induced apoptotic death of CD8 $+\mathrm{T}$ cells. Blood. 2003;101(7):2711-20.

20. Chattopadhyay PK, Betts MR, Price DA, Gostick E, Horton H, Roederer M, et al. The cytolytic enzymes granyzme $A$, granzyme $B$, and perforin: expression patterns, cell distribution, and their relationship to cell maturity and bright CD57 expression. J Leukoc Biol. 2009;85(1):88-97.

21. Hayhoe RP, Henson SM, Akbar AN, Palmer DB. Variation of human natural killer cell phenotypes with age: identification of a unique KLRG1-negative subset. Hum Immunol. 2010;71(7):676-81.

22. Hong HS, Eberhard JM, Keudel P, Bollmann BA, Ballmaier M, Bhatnagar N, et al. HIV infection is associated with a preferential decline in less-differentiated CD56dim CD16 + NK cells. J Virol. 2010;84(2):1183-8.

23. Lanier LL, Le AM, Phillips JH, Warner NL, Babcock GF. Subpopulations of human natural killer cells defined by expression of the Leu-7 (HNK-1) and Leu-11 (NK-15) antigens. J Immunol. 1983;131(4):1789-96. 
24. Nagler A, et al. Comparative studies of human FcRIll-positive and negative natural killer cells. $J$ Immunol. 1989;143(10):3183-91.

25. Abo T, Miller CA, Balch CM. Characterization of human granular lymphocyte subpopulations expressing HNK-1 (Leu-7) and Leu-11 antigens in the blood and lymphoid tissues from fetuses, neonates and adults. Eur J Immunol. 1984;14(7):616-23.

26. Krishnaraj R, Svanborg A. Preferential accumulation of mature NK cells during human immunosenescence. J Cell Biochem. 1992;50(4):386-91.

27. Le Garff-Tavernier M, Béziat V, Decocq J, Siguret V, Gandjbakhch F, Pautas E, et al. Human NK cells display major phenotypic and functional changes over the life span. Aging Cell. 2010;9(4):527-35.

28. Chen VC, Couture SM, Ye J, Lin Z, Hua G, Huang HI, et al. Scalable GMP compliant suspension culture system for human ES cells. Stem Cell Res. 2012;8(3):388-402.

29. Dakhore S, Nayer B, Hasegawa K. Human Pluripotent Stem Cell Culture: Current Status, Challenges, and Advancement. Stem Cells Int. 2018;2018:7396905.

30. Robinton DA, Daley GQ. The promise of induced pluripotent stem cells in research and therapy. Nature. 2012;481(7381):295-305.

31. Zhao T, Zhang ZN, Rong Z, Xu Y. Immunogenicity of induced pluripotent stem cells. Nature. 2011;474(7350):212-5.

32. Kim D, Kim CH, Moon JI, Chung YG, Chang MY, Han BS, et al. Generation of human induced pluripotent stem cells by direct delivery of reprogramming proteins. Cell Stem Cell. 2009;4(6):472-6.

33. Warren L, Manos PD, Ahfeldt T, Loh YH, Li H, Lau F, et al. Highly efficient reprogramming to pluripotency and directed differentiation of human cells with synthetic modified mRNA. Cell Stem Cell. 2010;7(5):618-30.

34. Park IH, Zhao R, West JA, Yabuuchi A, Huo H, Ince TA, et al. Reprogramming of human somatic cells to pluripotency with defined factors. Nature. 2008;451(7175):141-6.

35. Eminli S, Utikal J, Arnold K, Jaenisch R, Hochedlinger K. Reprogramming of neural progenitor cells into induced pluripotent stem cells in the absence of exogenous Sox 2 expression. Stem Cells. 2008;26(10):2467-74.

36. Kim JB, Zaehres H, Wu G, Gentile L, Ko K, Sebastiano V, et al. Pluripotent stem cells induced from adult neural stem cells by reprogramming with two factors. Nature. 2008;454(7204):646-50.

37. Hanna J, Markoulaki S, Schorderet P, Carey BW, Beard C, Wernig M, et al. Direct reprogramming of terminally differentiated mature B lymphocytes to pluripotency. Cell. 2008;133(2):250-64.

38. Sun N, Panetta NJ, Gupta DM, Wilson KD, Lee A, Jia F, et al. Feeder-free derivation of induced pluripotent stem cells from adult human adipose stem cells. Proc Natl Acad Sci U S A. 2009;106(37):15720-5.

39. Maherali N, Ahfeldt T, Rigamonti A, Utikal J, Cowan C, Hochedlinger K. A high-efficiency system for the generation and study of human induced pluripotent stem cells. Cell Stem Cell. 2008;3(3):340-5. 
40. Pei D, Shu X, Gassama-Diagne A, Thiery JP. Mesenchymal-epithelial transition in development and reprogramming. Nat Cell Biol. 2019;21(1):44-53.

41. Li X, Pei D, Zheng H. Transitions between epithelial and mesenchymal states during cell fate conversions. Protein Cell. 2014;5(8):580-91.

42. Shu $X$, Pei D. The function and regulation of mesenchymal-to-epithelial transition in somatic cell reprogramming. Curr Opin Genet Dev. 2014;28:32-7.

43. Ni A, Wu MJ, Nakanishi Y, Chavala SH. Facile and efficient reprogramming of ciliary body epithelial cells into induced pluripotent stem cells. Stem Cells Dev. 2013;22(18):2543-50.

44. Wang L, Wang L, Huang W, Su H, Xue Y, Su Z, et al. Generation of integration-free neural progenitor cells from cells in human urine. Nat Methods. 2013;10(1):84-9.

45. Sachamitr P, Hackett S, Fairchild PJ. Induced pluripotent stem cells: challenges and opportunities for cancer immunotherapy. Front Immunol. 2014;5:176.

46. Dillman RO, Duma CM, Ellis RA, Cornforth AN, Schiltz PM, Sharp SL, et al. Intralesional lymphokineactivated killer cells as adjuvant therapy for primary glioblastoma. J Immunother. 2009;32(9):914-9.

47. Tonn T, Schwabe D, Klingemann HG, Becker S, Esser R, Koehl U, et al. Treatment of patients with advanced cancer with the natural killer cell line NK-92. Cytotherapy. 2013;15(12):1563-70.

48. Zeng J, Tang SY, Toh LL, Wang S. Generation of "Off-the-Shelf" Natural Killer Cells from Peripheral Blood Cell-Derived Induced Pluripotent Stem Cells. Stem Cell Reports. 2017;9(6):1796-812.

\section{Figures}


A

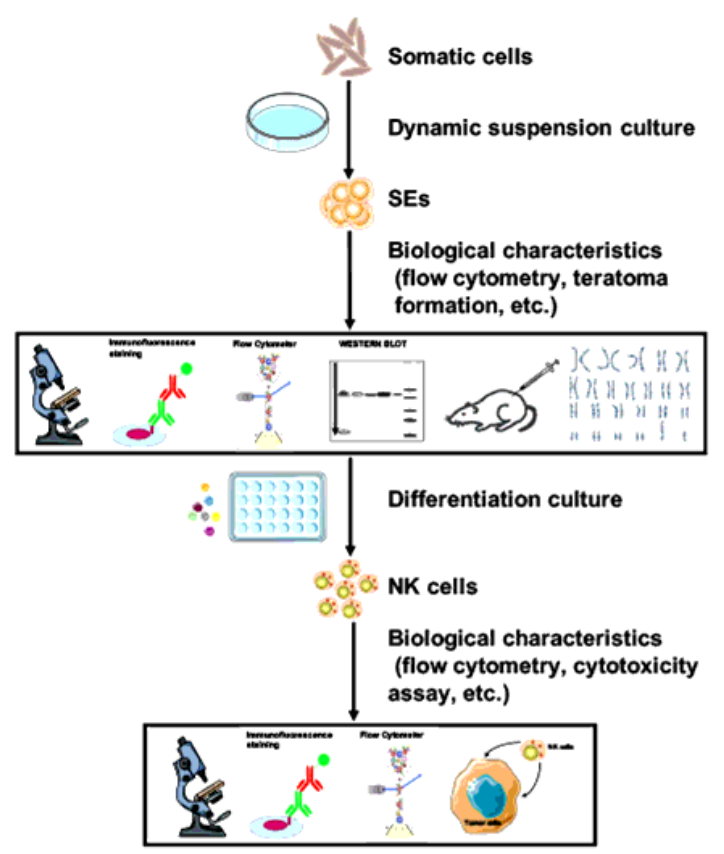

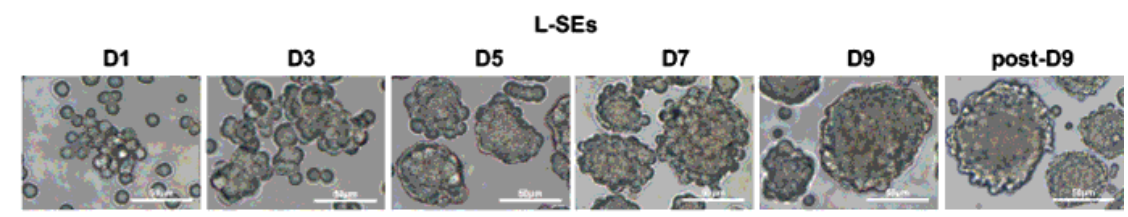

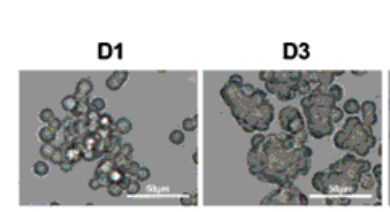

D5

H-SES

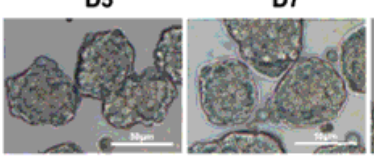

W-SEs

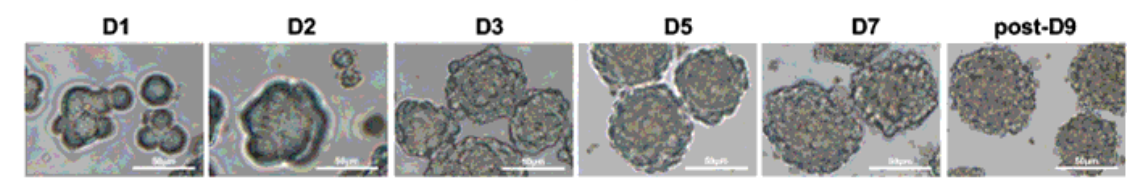

C
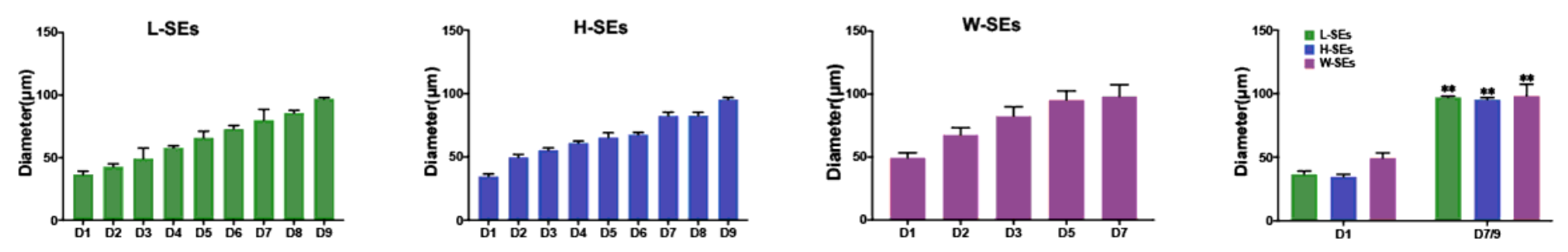

Figure 1

iPSCs in the form of SEs generated from somatic cells by using dynamic suspension culture. a A twostep protocol to produce NK cells from somatic cells. First, dynamic suspension culture was utilized to generate SEs formation and multi-method was used to assay the biological characteristics of SES. Second, SEs were treated with cytokines to induce NK cells and multi-method was used to assay the biological characteristics of NK cells. b Changes of morphology during L-SEs, H-SEs, and W-SEs formation. iPSCs can be observed as SEs that were compact sphere and defined by clear borders. $\mathrm{C}$ Changes of diameter in L-SEs, H-SEs, and W-SEs during dynamic suspension culture. Diameter of SEs increased by degrees and was significant differences between day 1 and day $7 / 9$. Data are represented as mean $\pm \operatorname{SEM}(n=3)$. ** $P<0.01$. Scale bars $50 \mu \mathrm{m}$ 
A

L-SEs

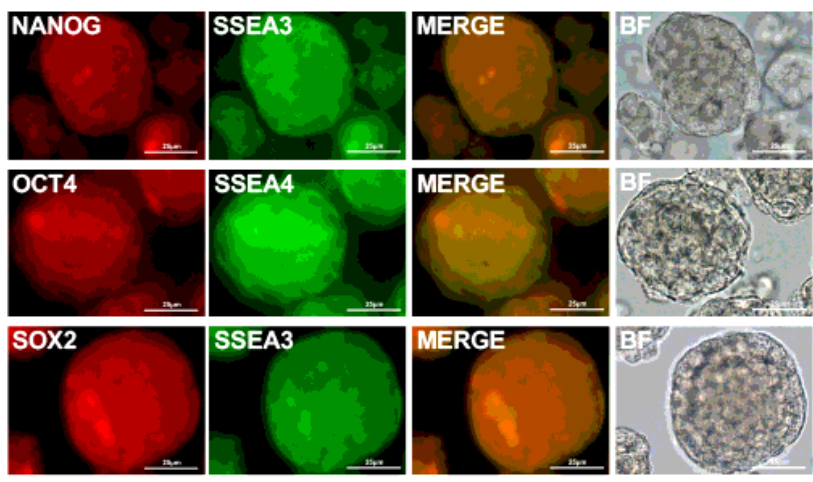

C

\section{W-SEs}
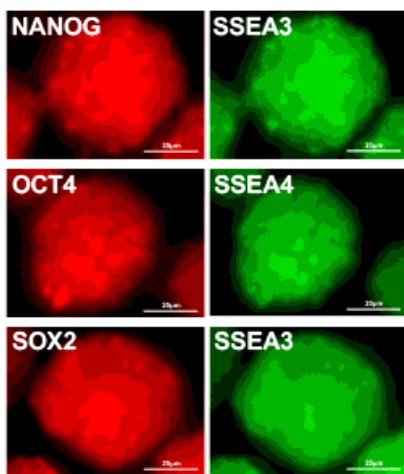

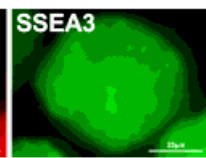

B

H-SEs

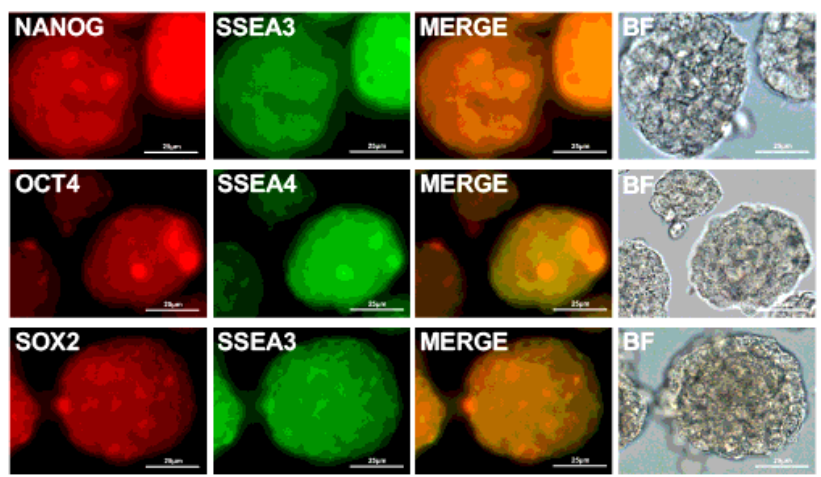

D

\section{L-SEs, H-SEs and W-SEs expressed}

pluripotent-associated marker

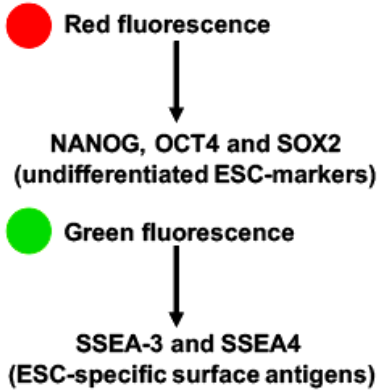

Figure 2

Pluripotent-associated marker expression of SEs by immunofluorescence staining. a-d L-SEs (a), H-SEs (b), and W-SEs (c) expressed undifferentiated ESC-markers, such as NANOG, OCT4, and SOX2 (red) and ESC-specific surface antigens, including SSEA-3 and SSEA4 (green) (d). Scale bars $25 \mu \mathrm{m}$

A

L-SEs
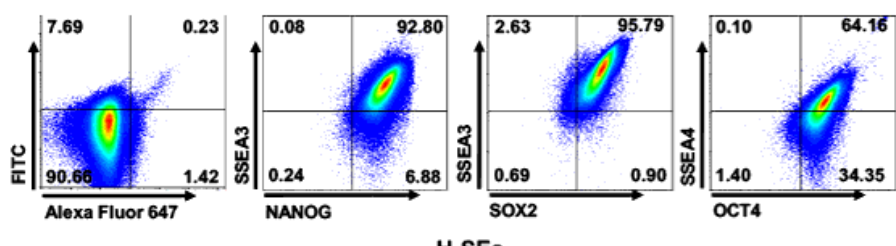

H-SES
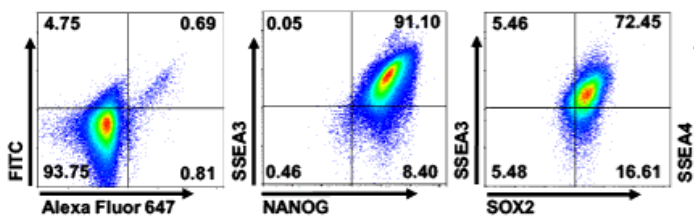

W-SES
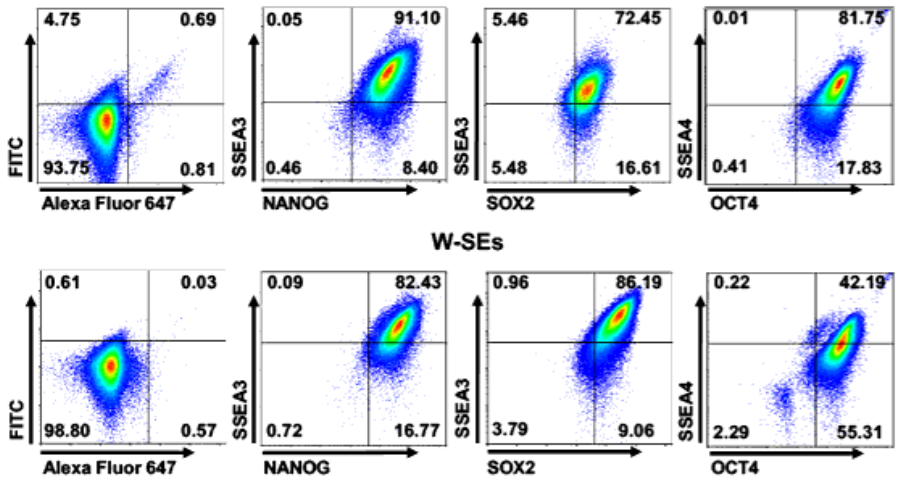

B

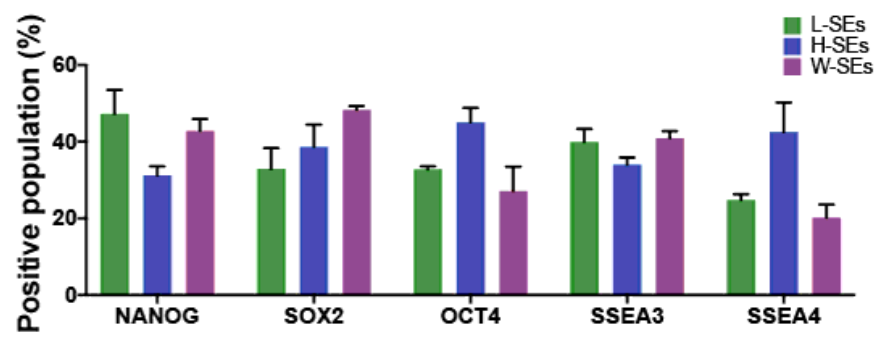

C

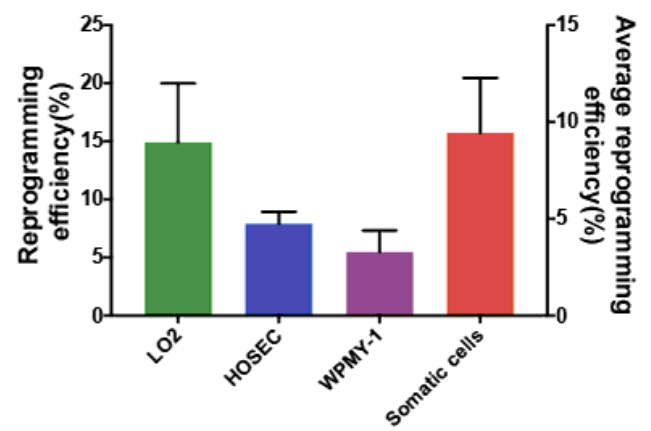




\section{Figure 3}

Pluripotent-associated marker analysis of SEs by flow cytometry. a Two-color flow cytometry revealed that L-SEs, H-SEs, and W-SEs expressed pluripotent-associated marker NANOG, OCT4, SOX2, SSEA-3, and SSEA4. b The analysis of flow cytometry showed that the expression levels of NANOG, OCT4, SOX2, SSEA-3, and SSEA-4 were comparable among L-SEs, H-SEs, and W-SEs. Data are represented as mean \pm SEM $(n=3)$. $c$ The reprogramming efficiency of LO2, HOSEC, and WPMY-1 was no significant differences and the average reprogramming efficiency of somatic cells was $9.44 \pm 2.83 \%$ by using dynamic suspension culture system. Data are represented as mean $\pm \operatorname{SEM}(n=3)$.

A

B

\section{W-SEs H-SEs L-SEs}

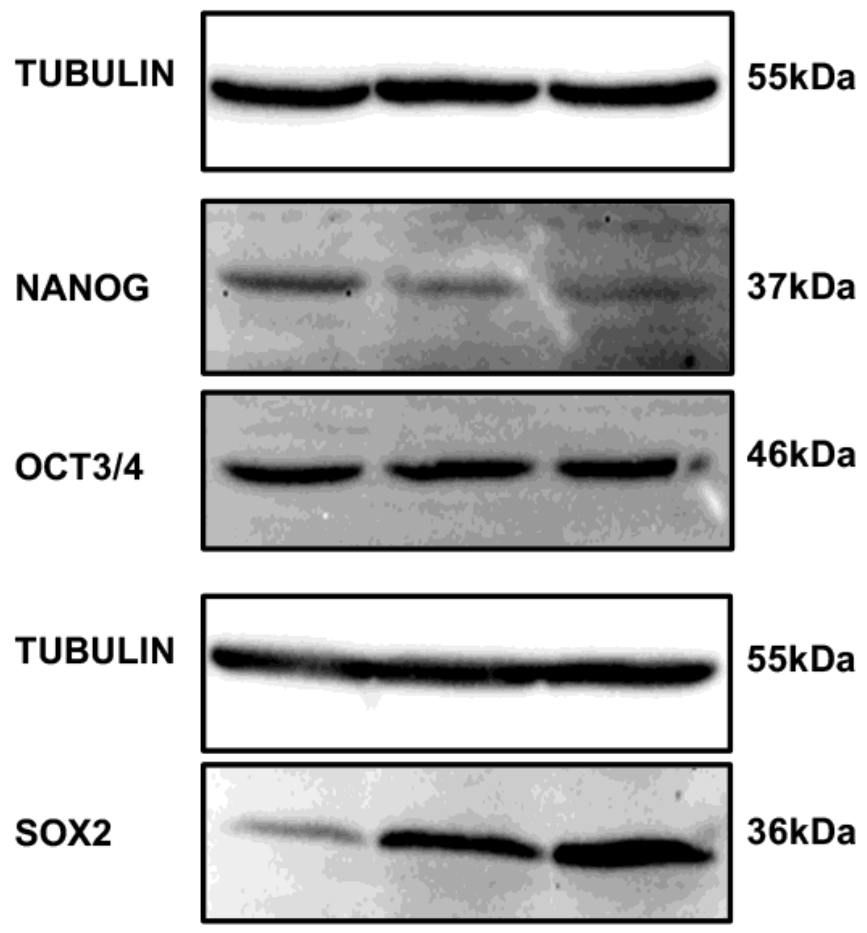

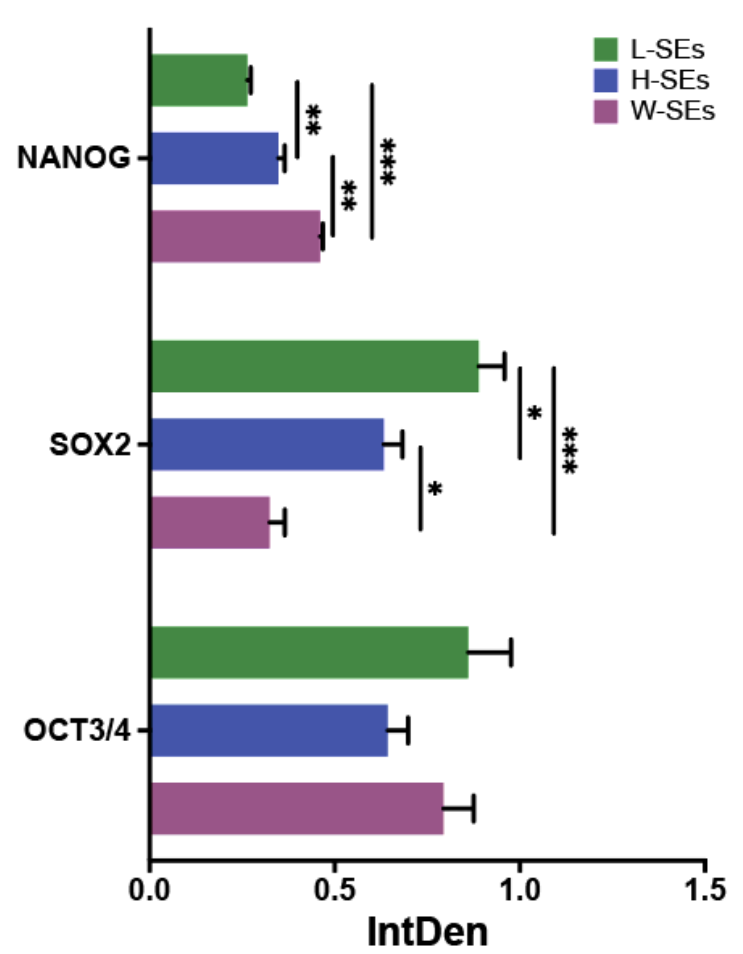

\section{Figure 4}

Pluripotent-associated marker analysis of SEs by western blot. a Western blot analysis revealed that LSEs, H-SEs, and W-SEs expressed NANOG, SOX2, and OCT3/4. b Protein levels of NANOG, SOX2, and OCT3/4 of SEs. Protein level of NANOG in H-SEs was higher significantly than in L-SEs. Protein level of NANOG in W-SEs was higher significantly than in L-SEs and H-SEs. Protein level of SOX2 in H-SEs was higher significantly than in W-SEs. Protein level of SOX2 in L-SEs was higher significantly than in H-SEs and W-SEs. Protein level of OCT3/4 was comparable among L-SEs, H-SEs, and W-SEs. Data are represented as mean \pm SEM $(n=3)$. ${ }^{*} P<0.05,{ }^{*} P<0.01$, $* \star * ~ P<0.001$. 


\section{A}

\section{endoderm}

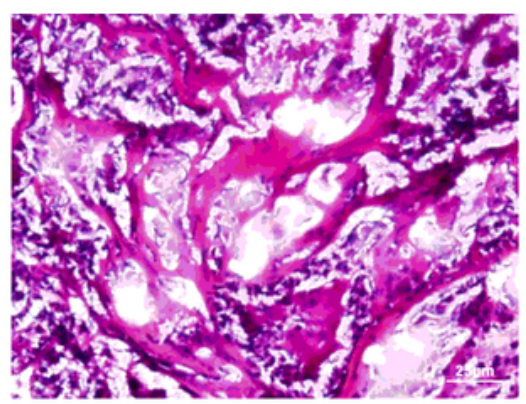

mesoderm

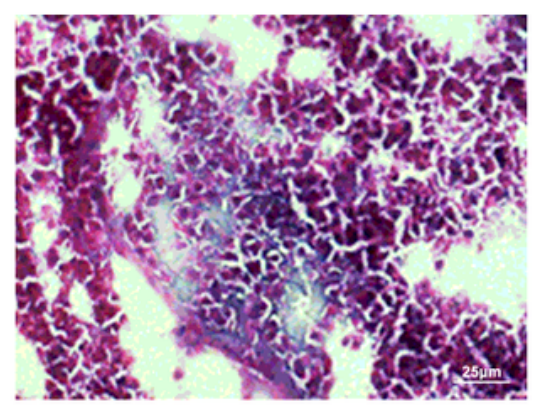

ectoderm

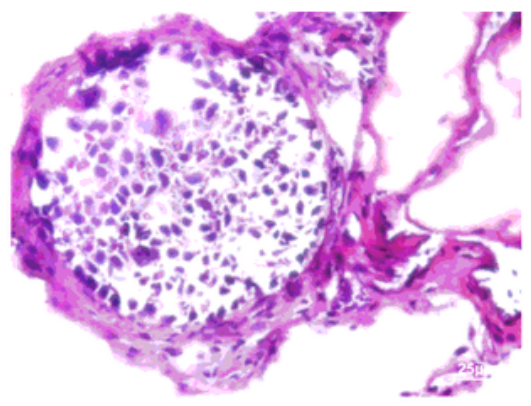

B

\section{L-SEs}

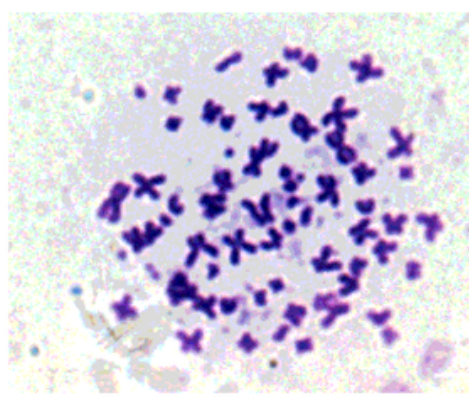

LO2

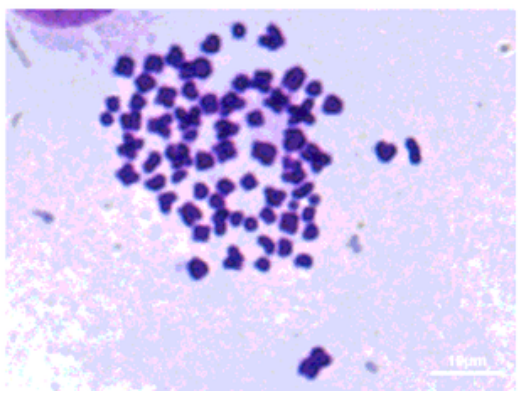

\section{H-SES}

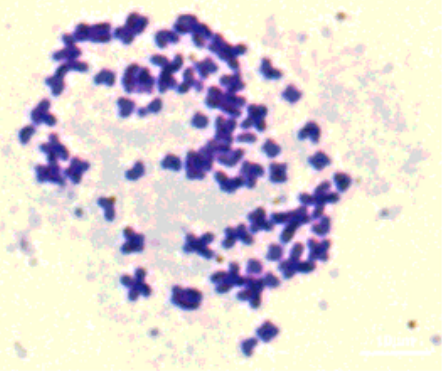

HOSEC

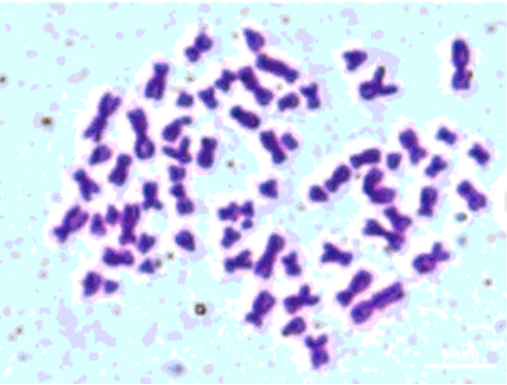

\section{W-SEs}

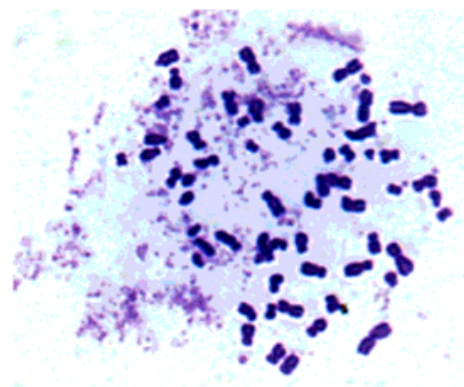

WPMY-1

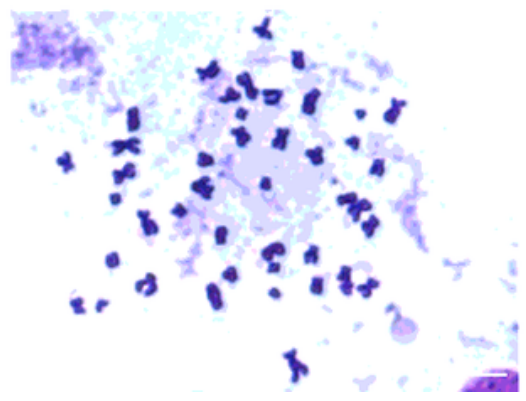

Figure 5

Pluripotency and chromosomal analysis of SEs by teratoma formation and karyotype analysis. a Hematoxylin and eosin staining revealed that teratoma contained all three germ layers tissues. Scale bars $25 \mu \mathrm{m}$ b Karyotype analysis revealed that chromosomal integrity of L-SEs, H-SEs, W-SEs, and somatic cells. Scale bars $10 \mu \mathrm{m}$ 


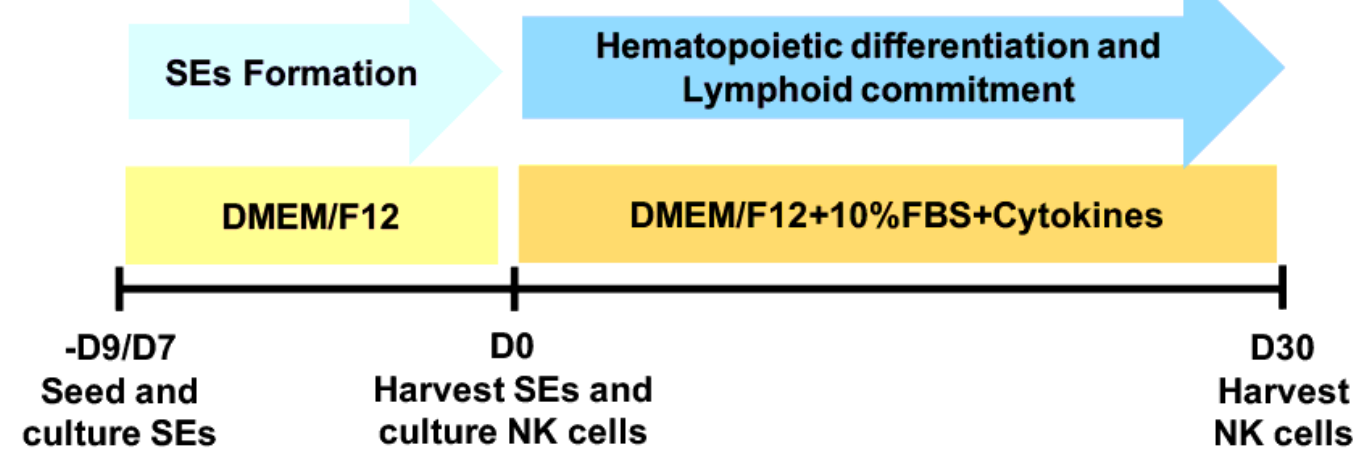

B

\section{L-SEs-NK}
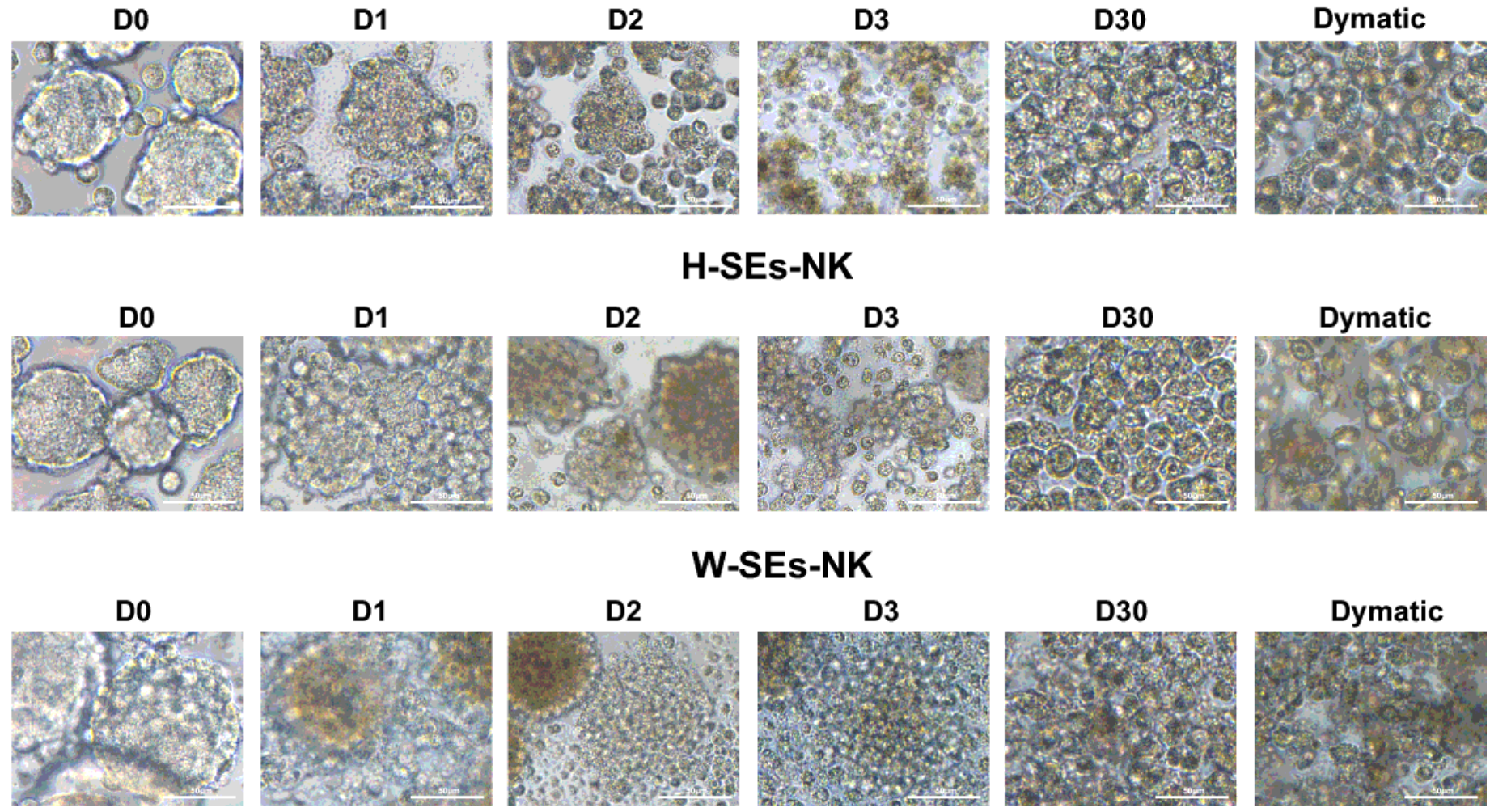

Figure 6

Induction of NK cells from SEs. a Time schedule of formation of SEs-NK cells. b Changes of morphology during SEs-NK cells formation. The spherical shape of L-SEs, H-SEs, and W-SEs was not maintained, and some single cells appeared during the first three days. After 30 days, there were a large number of lymphoid cells. Scale bars $50 \mu \mathrm{m}$ 
A

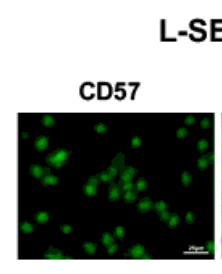

L-SEs-NK

BF

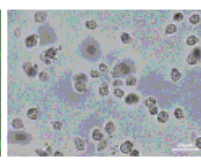

H-SEs-NK

CD57

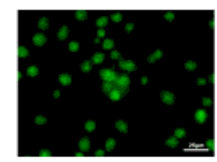

BF

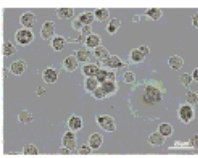

W-SEs-NK

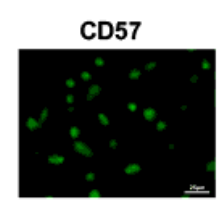

LO2

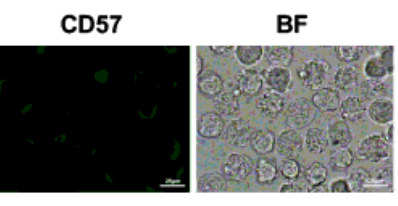

HOSEC

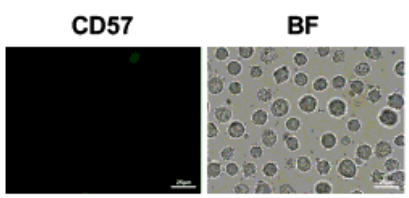

WPMY-1

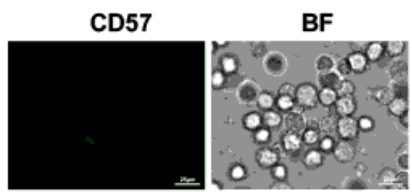

B
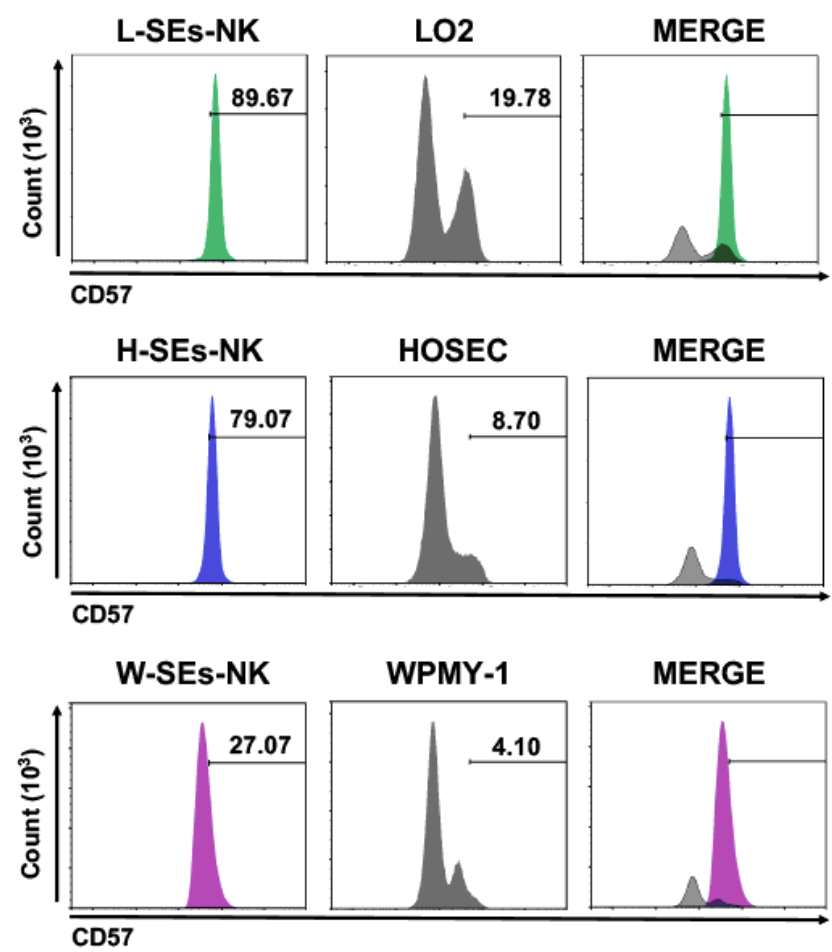

Figure 7

NK cell marker analysis of SEs-NK cells by immunofluorescence staining and flow cytometry. a Immunostaining revealed that CD57 (green) was expressed on L-SEs-NK, H-SEs-NK, and W-SEs-NK cells, but not on three somatic cells. b Flow cytometry revealed that the expression levels of CD57 on L-SEs-NK, H-SEs-NK, and W-SEs-NK cells was higher than that on the three somatic cells. Scale bars $25 \mu \mathrm{m}$ 


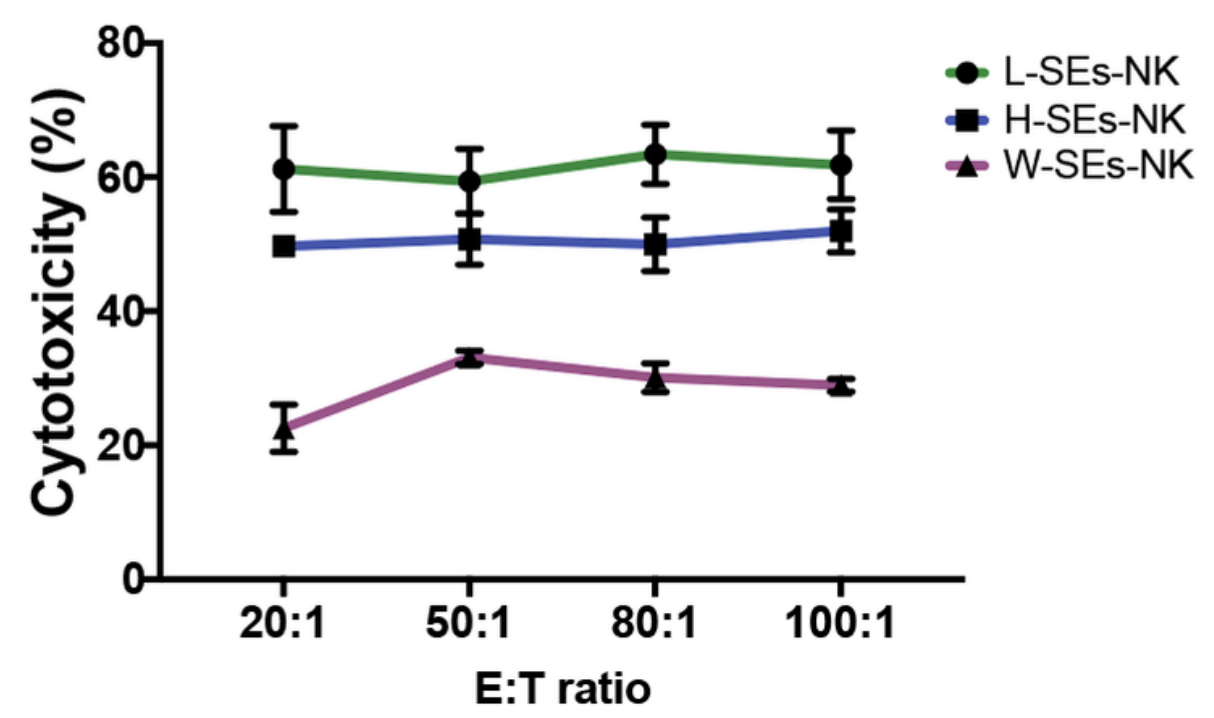

B

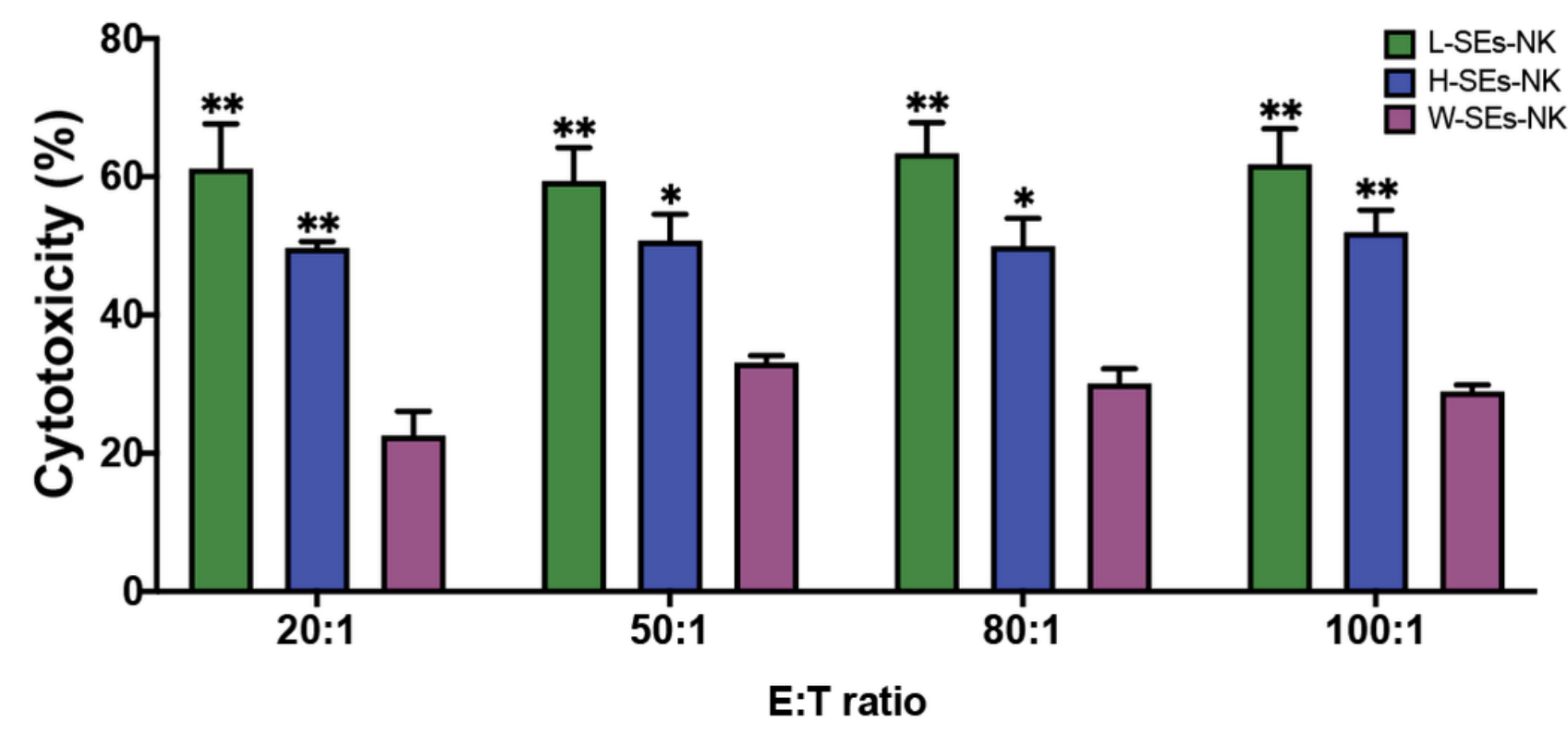

Figure 8

Cytotoxicity assay of SEs-NK cells. a Cytotoxicity assay of L-SEs-NK, H-SEs-NK, and W-SEs-NK cells against the leukemic cell line K562. Data are represented as mean \pm SEM $(n=3)$. b Comparation of LSEs-NK, H-SEs-NK, and W-SEs-NK cells against K562 cells at E:T ratios of 20:1, 50:1, 80:1, and 100:1. Significant differences between L-SEs-NK and W-SEs-NK cells at different E:T ratios are presented. Significant differences between H-SEs-NK and W-SEs-NK cells at different E:T ratios are presented. Data are represented as mean \pm SEM $(n=3)$. ${ }^{*} P<0.05, * * P<0.01$. 


\section{Supplementary Files}

This is a list of supplementary files associated with this preprint. Click to download.

- Additionalfile1.pdf

- Additionalfile2.pdf

- Additionalfile3.pdf

- Additionalfile4.pdf

- Additionalfile5.pdf

- Additionalfile6.pdf 